\title{
A randomized trial to evaluate the impact of exercise-based cardiac rehabilitation for the prevention of chemotherapy-induced cardiotoxicity in patients with breast cancer: ONCORE study protocol
}

\author{
Estíbaliz Díaz-Balboa ${ }^{1,2,3^{*}}$ (D) Violeta González-Salvado ${ }^{1,2}$, Beatriz Rodríguez-Romero ${ }^{4}$, \\ Amparo Martínez-Monzonís ${ }^{1,2}$, Milagros Pedreira-Pérez ${ }^{1,2}$, Patricia Palacios-Ozores ${ }^{2,5}$, Rafael López-López ${ }^{2,5}$, \\ Carlos Peña-Gil ${ }^{1,2}$ and José R. González-Juanatey ${ }^{1,2}$
}

\begin{abstract}
Background: Anthracyclines and monoclonal antibodies against human epidermal growth factor receptor-2 (HER2) are frequently used to treat breast cancer but they are associated with risk of developing cardiotoxicity. Implementation of cardioprotective strategies as part of breast cancer treatment are needed. To date, a limited number of studies have examined the effectiveness of cardiac rehabilitation programs or exercise programs in the prevention of cardiotoxicity through an integral assessment of cardiac function. The ONCORE study proposes an exercise-based cardiac rehabilitation program as a non-pharmacological tool for the management of chemotherapy-induced cardiotoxicity.

Methods: The study protocol describes a prospective, randomized controlled trial aimed to determine whether an intervention through an exercise-based CR program can effectively prevent cardiotoxicity induced by anthracyclines and/or anti-HER2 antibodies in women with breast cancer. Three hundred and forty women with breast cancer at early stages scheduled to receive cardiotoxic chemotherapy will be randomly assigned (1:1) to participation in an exercise-based CR program (intervention group) or to usual care and physical activity recommendation (control group). Primary outcomes include changes in left ventricular ejection fraction and global longitudinal strain as markers of cardiac dysfunction assessed by transthoracic echocardiography. Secondary outcomes comprise levels of cardiovascular biomarkers and cardiopulmonary function through peak oxygen uptake determination, physical performance and psychosocial status. Supervised exercise program-related outcomes including safety, adherence/ compliance, expectations and physical exercise in- and out-of-hospital are studied as exploratory outcomes. Transthoracic echocardiography, clinical test and questionnaires will be performed at the beginning and two weeks after completion of chemotherapy.
\end{abstract}

\footnotetext{
*Correspondence: estibaliz.diaz@udc.es

${ }^{1}$ Cardiology Department, Centro de Investigación Biomédica en Red de Enfermedades Cardiovasculares (CIBERCV), University Clinical Hospital of Santiago de Compostela (SERGAS), A Choupana s/n, 15706 Santiago de Compostela, A Coruña, Spain

Full list of author information is available at the end of the article
} original author(s) and the source, provide a link to the Creative Commons licence, and indicate if changes were made. The images or other third party material in this article are included in the article's Creative Commons licence, unless indicated otherwise in a credit line to the material. If material is not included in the article's Creative Commons licence and your intended use is not permitted by statutory regulation or exceeds the permitted use, you will need to obtain permission directly from the copyright holder. To view a copy of this licence, visit http://creativecommons.org/licenses/by/4.0/. The Creative Commons Public Domain Dedication waiver (http://creativeco mmons.org/publicdomain/zero/1.0/) applies to the data made available in this article, unless otherwise stated in a credit line to the data. 
Discussion: The growing incidence of breast cancer and the risk of cardiotoxicity derived from cancer treatments demand adjuvant cardioprotective strategies. The proposed study may determine if an exercise-based CR program is effective in minimizing chemotherapy-induced cardiotoxicity in this population of women with early-stage breast cancer. The proposed research question is concrete, with relevant clinical implications, transferable to clinical practice and achievable with low risk.

Trial registration ClinicalTrials.gov Identifier: NCT03964142. Registered on 28 May 2019. Retrospectively registered. https://clinicaltrials.gov/ct2/show/NCT03964142

Keywords: Breast cancer, Cardiotoxicity, Cardiac rehabilitation, Exercise, Cardio-oncology, Anthracyclines, HER2 overexpression

\section{Background}

Survival in breast cancer has improved due to advances in early detection and treatment, leading to reduced breast cancer-related mortality [1]. However, many of the current cancer therapies are linked to multiple side effects. Of these treatments, chemotherapy has been associated with adverse cardiovascular events, such as decreased cardiac function, heart failure, coronary artery disease, arterial hypertension or thromboembolism [2, 3]. In fact, cardiovascular disease is the leading cause of death among women with early-stage breast cancer [4]. The absolute risk of dying from cardiovascular disease in breast cancer ranges from 1.6 to $10.4 \%$ [5], and it is higher than that of women from the general population $[5,6]$. Treatment using anthracyclines and monoclonal antibodies against human epidermal growth factor receptor 2 (HER2) has resulted in substantial improvements in survival, but these agents have been associated with the development of cardiotoxicity [7, 8]. Cardiotoxicity is defined by either a decrease in left ventricular ejection fraction (LVEF) above 10\% from baseline to a value of LVEF under 53\%, or a decrease in global longitudinal strain (GLS) deformation below 15\% from baseline value $[9,10]$. While transthoracic echocardiography (TTE) is the standard method to evaluate cardiotoxicity [9], comprehensive assessment of cardiac function may include other tests, such as biomarkers (troponin I and B-type natriuretic peptide) [11] and cardiopulmonary function measured by peak oxygen uptake (VO2). The latter is considered as a gold standard parameter, which may decrease by $5-26 \%$ during exposure to various treatment regimens [12]. The combination of global and specific measures of cardiac function allows identifying patients at risk of heart failure more accurately and initiating appropriate treatment $[13,14]$. To respond to this challenge, Cardio-Onco-Hematology $(\mathrm{COH})$ units for early diagnosis and treatment have emerged $[12,15]$.

Exercise programs are increasingly being recognized as an effective strategy to counteract adverse effects of cancer therapy [16-19]. While there is extensive evidence on the benefits of exercise to mitigate side effects such as fatigue [20] or to improve quality of life [21], evidence on the cardioprotective effects of exercise in breast cancer is still limited [4, 22-24]. In addition to exercise programs, cardiac rehabilitation (CR) programs have proven beneficial to lessen the consequences of cardiovascular disease and reduce the risk of future events [25]. CR programs perform a comprehensive evaluation of cardiovascular risk and a multi-faceted intervention including dietary counselling, weight and blood pressure control, lipid profile optimisation, smoking cessation, psychosocial support, adherence to medical treatment and structured exercise therapy as a central component [26]. Participation in structured CR programs is associated with reduced mortality after an acute coronary event [27], and also with improvement of event-free survival in patients with heart failure [28]. Nonetheless, programs based on CR are not yet standardized within breast cancer care [29].

The main aim of this study is to determine whether an intervention through an exercise-based CR program could effectively prevent cardiotoxicity induced by anthracyclines and/or anti-HER2 antibodies in women with breast cancer at early stages, as compared with usual care and physical activity recommendation. Secondary aims are to determine the effect of the intervention on other cardiac function parameters (cardiopulmonary function assessed by peak $\mathrm{VO} 2$ and biomarkers), physical performance outcomes (e.g., body composition, limb strength and upper limb function); and psychometric and lifestyle outcomes (e.g., quality of life, physical exercise, diet pattern). Furthermore, safety of the intervention and supervised exercise program outcomes (adherence-compliance, expectations, satisfaction) are also assessed.

We hypothesize that an exercise-based CR program will be effective in preventing chemotherapy-induced cardiotoxicity in women with breast cancer at early stages, compared to usual care and physical exercise recommendation. Moreover, we hypothesize that patients in the intervention group will maintain or improve their physical performance and psychosocial status. 


\section{Methods}

This study protocol follows the SPIRIT 2013 [30] recommendations, an additional checklist file shows this in more detail (Additional file 1). This is a two-arm, prospective, randomized controlled trial comparing an exercise-based CR program (experimental group) to usual care and physical activity recommendation (control group) in women receiving anthracycline and/or anti-HER2 antibodies for early breast cancer. The trial is conducted based on the coordinated work of the $\mathrm{COH}$ and the CR and Preventive Cardiology units at the University Clinical Hospital of Santiago de Compostela (Galicia, Spain). The design of the trial is shown in Fig. 1. The study has been approved by the Ethics Committee of Clinical Investigation of Galicia (CEIC reference number 2018/083). Financial support for this work is provided by the Carlos III Health Institute (ISCIII) - PI17/01687, co-funded FEDER, through a competitive grant of the Healthcare Research Fund, under the Strategic Health Action call for proposals.

\section{Study duration and timing}

The study timeline is depicted in Fig. 2. Patient recruitment and data collection started in August 2018. The inclusion and baseline assessment of patients is carried out progressively as they start treatment with cardiotoxic chemotherapy including anthracyclines and/or antiHER2 antibodies, adjusting to the reality of routine clinical practice. The intervention lasts until the last patient recruited finishes the cardiotoxic treatment, between 6 and 12 months, depending on whether this includes anti-HER2 antibodies. Post-treatment assessment is performed two weeks after the last cycle of cardiotoxic chemotherapy including anti-HER2 antibodies. Data collection is expected to be completed in March 2022 when all patients in the study will have completed their respective treatments and undergone post-treatment assessment.

\section{Recruitment and inclusion}

The study population consists of female patients aged between 18 and 70 years with a first diagnosis of breast cancer at early stages (I, II, III), who are planned to receive anthracyclines and/or anti-HER2 antibodies (trastuzumab and pertuzumab), without contraindications to join a CR program or to complete the scheduled visits and providing written consent to participate. If patients are referred once chemotherapy has been initiated, only those who have received less than $20 \%$ sessions of the cardiotoxic treatment planned will be eligible for the study. Inclusion and exclusion criteria are detailed in Table 1. Potential participants are detected in daily practice among patients referred to the $\mathrm{COH}$ unit to undergo the established protocol to identify cardiotoxicity (clinical, laboratory and echocardiographic follow-up assessment). $\mathrm{COH}$ specialists are responsible for initial screening, providing information about the study and offering informed consent to patients.

\section{Randomization}

After signing the informed consent and undergoing initial assessment, patients are randomized to the experimental group (EG) or the control group (CG) by simple randomization performed by an external person using a computer-generated number sequence. The physiotherapist is responsible for informing each patient of the follow-up modality. The EG will join an exercise-based CR program, while the CG will receive conventional management and physical activity recommendation.

\section{Intervention}

\section{Experimental group: exercise-based CR program}

The intervention in the EG consists of a supervised exercise-based CR program undergone during chemotherapy, which can be administrated before (neoadjuvant) and/or after (adjuvant) surgery. The supervised exercise program takes place at the hospital CR room and is guided and supervised by a physiotherapist, in groups of 7 participants twice a week. Each 1-h session will comprise four parts.

- First part: 15 min of breathing, mobility and balance exercises developed through 8 exercise with 1 set of 7-10 repetitions.

- Second part: 15 min of strength exercises consisting of 4 bodyweight exercises with 2 sets of 7-10 repetitions and 5 resistance bands exercises with 2 sets of 7-10 repetitions.

- Third part: 25 min of continuous or interval exercise in the aerobic-anaerobic transition zone using a cycle ergometer or a treadmill. The patients are monitored with telemetry (Ergoline ERS 2, Germany). A target of $50-85 \%$ of heart rate reserve, according to the cardiopulmonary exercise testing (CPET), will be aimed. This objective may be adjusted according to patient's perceived exertion to rate subjective fatigue values between 3 and 7 (Borg Scale 0-10) and the physiotherapist's criteria.

- Fourth part: 5 min of flexibilization including 6 types of exercises for the major muscle groups. 


\section{Enrolment:}

Screening of women with breast cancer who will be prescribed cardiotoxic treatment (anthracyclines and/or anti-HER2 antibodies) at the Cardio-Onco-Hematologic unit

Patients not eligible

Baseline assessment:

- Cardiotoxicity protocol detection (clinical evaluation, blood tests and echocardiography)

- Functional assessment (questionnaires, cardiopulmonary exercise testing, clinical test)

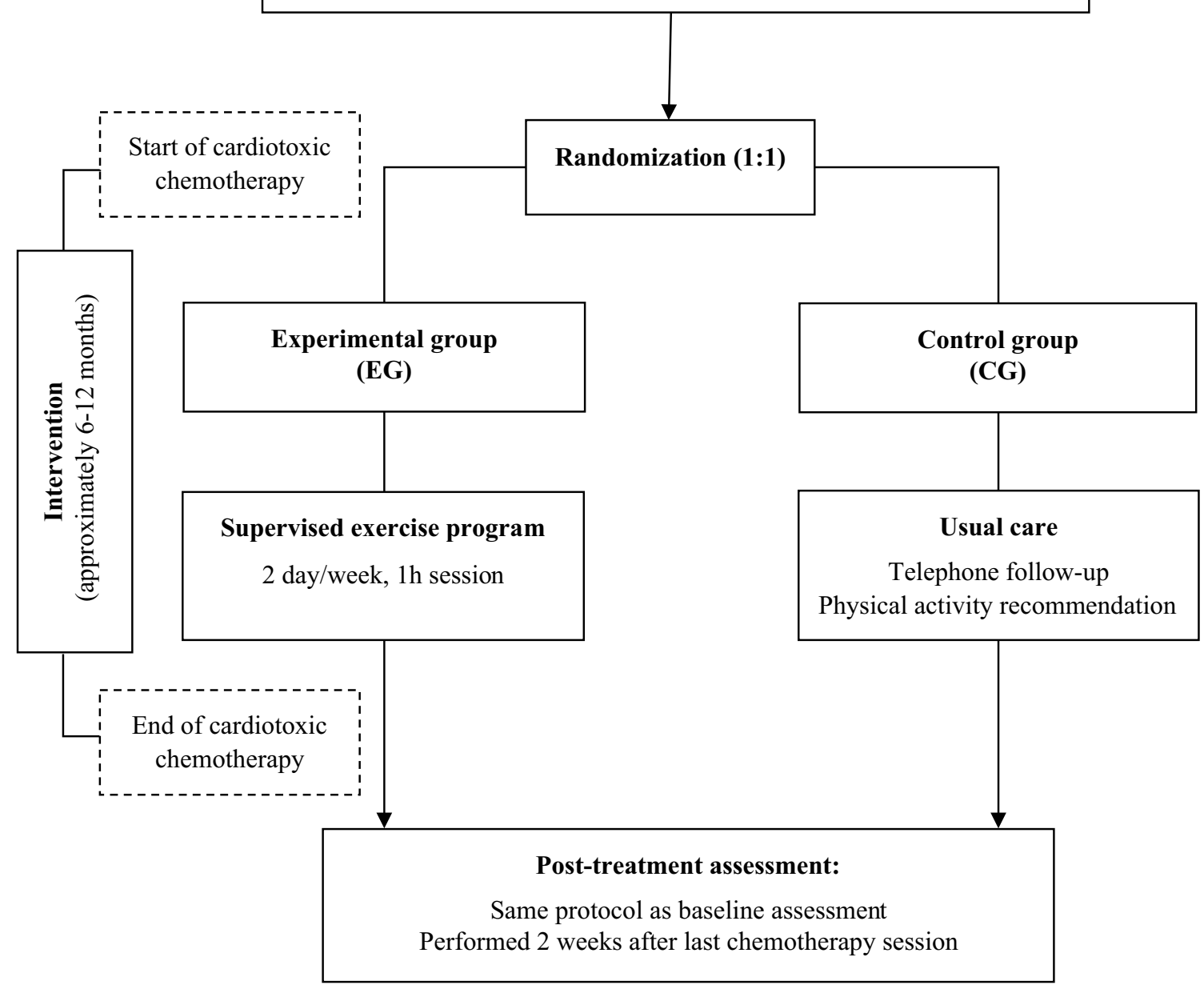

Fig. 1 Flowchart ONCORE design 


\begin{tabular}{|c|c|c|c|c|}
\hline \multirow[b]{2}{*}{ Phase } & \multirow[b]{2}{*}{ Location } & \multicolumn{3}{|c|}{$\begin{array}{l}\text { Cardiotoxic treatment with anthracyclines and/or anti-HER2 antibodies } \\
\text { (ranging 6-12 months) }\end{array}$} \\
\hline & & $\begin{array}{c}\text { Before } \\
\text { treatment }\end{array}$ & $\begin{array}{c}\text { During } \\
\text { treatment }\end{array}$ & $\begin{array}{c}\text { After } \\
\text { treatment } * *\end{array}$ \\
\hline Enrolment & $\mathrm{COHU}$ & $\mathrm{X}$ & & \\
\hline $\begin{array}{c}\text { Baseline } \\
\text { assessment }\end{array}$ & $\begin{array}{l}\text { COHU } \\
\text { CRPCU }\end{array}$ & $\mathrm{X}$ & & \\
\hline Intervention & CRPCU & & $\mathrm{X}$ & \\
\hline $\begin{array}{l}\text { Post-treatment } \\
\text { assessment }\end{array}$ & $\begin{array}{l}\text { COHU } \\
\text { CRPCU }\end{array}$ & & & $X$ \\
\hline $\begin{array}{l}* \text { Only pati } \\
\mathrm{COHU} \\
\text { Fig. } 2 \text { Study timeline }\end{array}$ & $\begin{array}{l}\text { ts receiving }< \\
* * \text { final ass } \\
\text { dio-onco-her }\end{array}$ & $\begin{array}{l}0 \% \text { sessions of } \mathrm{t} \\
\text { sment will take } \\
\text { atology unit; } C R\end{array}$ & $\begin{array}{l}\text { oxic treatmen } \\
\text { ast chemothe } \\
\text { litation and p }\end{array}$ & $\begin{array}{l}\text { legible } \\
\text { Init }\end{array}$ \\
\hline
\end{tabular}

Table 1 Inclusion and exclusion criteria

\begin{tabular}{|c|c|}
\hline Inclusion criteria & Exclusion criteria \\
\hline Woman aged $>18$ years and $<70$ years & Patients with previous history of heart disease or heart failure \\
\hline First diagnosis of breast cancer at early stages $(I, I I, I I)$ & $\begin{array}{l}\text { Left ventricular dysfunction (left ventricular ejection fraction }<53 \% \text { ) at } \\
\text { baseline }\end{array}$ \\
\hline $\begin{array}{l}\text { Patients who will receive adjuvant or neoadjuvant treatment with curative } \\
\text { intention }\end{array}$ & Metastatic disease \\
\hline $\begin{array}{l}\text { Treatment with anthracyclines and/or anti-HER-2 antibodies (trastuzumab } \\
\text { and pertuzumab) }\end{array}$ & Patients carrying an implantable cardioverter defibrillator \\
\hline $\begin{array}{l}\text { Possibility of completing a cardiac rehabilitation program and pro- } \\
\text { grammed visits }\end{array}$ & Patients with physical or mental limitation to carry out an exercise program \\
\hline \multirow[t]{2}{*}{ Providing written informed consent } & Refuse to participate \\
\hline & Overcome $20 \%$ of cardiotoxic treatment \\
\hline
\end{tabular}

Patients in the EG are offered to request an appointment with the CR psychologist if needed. Sessions are held individually for about one hour.

\section{Control group: usual care and physical activity recommendation}

Participants in the CG receive conventional management with general physical activity recommendation. The physiotherapist performs a clinical interview by telephone every two months until final assessment.

\section{Common intervention for both groups}

All patients receive the same supervision within the $\mathrm{COH}$ unit, every 2 months since the beginning of the treatment, including cardiotoxicity detection and cardiovascular risk factor control, consisting of dietary advice, smoking cessation, lipid profile, blood pressure control and cardiovascular medical treatment optimization. In addition, a practice guide with exercise recommendations and an exercise routine example is provided, as well as an exercise diary to register physical exercise (including date, minutes and type of exercise done) on a daily basis. Participants in both groups are encouraged and reminded of the importance of adherence to physical exercise.

\section{Criteria for drop out}

Criteria for discontinuing allocated interventions are participant's will to revoke participation in the study, 
worsening of oncological prognosis that prevents the continuation and absence of training sessions for unjustified reasons in the case of the EG (below $60 \%$ compliance).

\section{Study outcomes}

Outcomes measures and assessment methods are summarized in Table 2. Primary and secondary outcomes are assessed at the beginning and two weeks after completion of chemotherapy. Exploratory outcomes are collected post treatment. The $\mathrm{COH}$ specialists perform echocardiographic evaluation and scheduled blood

Table 2 Study outcome measure

\begin{tabular}{|c|c|c|c|}
\hline Domain & Measure & Assessment Method & Timepoint from chemotherapy \\
\hline \multicolumn{4}{|l|}{ Primary outcomes } \\
\hline \multirow[t]{2}{*}{ Cardiotoxicity } & Resting LVEF & TTE & Pre-post \\
\hline & Resting GLS & TTE & Pre-post \\
\hline \multicolumn{4}{|l|}{ Secondary outocomes } \\
\hline Cardiopulmonary function & VO2 peak & CPET & Pre-post \\
\hline \multirow[t]{3}{*}{ Biomarkers } & NT- proBNP & Blood test & Pre-post \\
\hline & Troponin I & Blood test & Pre-post \\
\hline & Hemoglobin & Blood test & Pre-post \\
\hline \multirow[t]{2}{*}{ Body composition } & $\%$ Body fat & Tanita & Pre-post \\
\hline & $\%$ Lean mass & Tanita & Pre-post \\
\hline \multirow[t]{4}{*}{ Anthropometrics } & Height & Height road & Pre \\
\hline & Weight & Tanita & Pre-post \\
\hline & Abdominal circumference & Tape measure & Pre-post \\
\hline & BMI & Database & Pre-post \\
\hline \multirow[t]{2}{*}{ Vital signs } & Heart rate & BPM & Pre-post \\
\hline & Blood pressure & BPM & Pre-post \\
\hline \multirow[t]{4}{*}{ Cardiovascular risk factors } & Dyslipidemia & Clinical history & Pre-post \\
\hline & Diabetes mellitus & Clinical history & Pre-post \\
\hline & Arterial hypertension & Clinical history & Pre-post \\
\hline & Smoking status & Clinical interview & Pre-post \\
\hline \multirow[t]{2}{*}{ Limb strength } & Lower limb strenght & 30-STS & Pre-post \\
\hline & Uper limb strenght & Handgrip dinamometry & Pre-post \\
\hline \multirow[t]{3}{*}{ Upper limb function in women undergone surgery } & Limb perimeters & Tape measure & Pre and/or post if surgery \\
\hline & Shoulder functional capacity & Goniometry & Pre and/or post if surgery \\
\hline & Shoulder pain and disability & SPADI & Pre and/or post if surgery \\
\hline \multirow[t]{4}{*}{ Psychometric and lifestyle questionnaires } & Anxiety and depression & HADS & Pre-post \\
\hline & Health-related quality of life & $\mathrm{FACT}-\mathrm{B}+4$ & Pre-post \\
\hline & Level of physical activity & GLTEQ & Pre-post \\
\hline & Adherence mediterranean diet & PREDIMED & Pre-post \\
\hline \multicolumn{4}{|l|}{ Exploratory outcomes } \\
\hline Physical exercise & $\begin{array}{l}\text { Minutes of In- (EG) and out- (both } \\
\text { groups) of-hospital dedicated } \\
\text { exercise }\end{array}$ & Exercise diary & Post \\
\hline Safety of the intervention & $\begin{array}{l}\text { Adverse events during exercise } \\
\text { and clinical serious adverse } \\
\text { events }\end{array}$ & $\begin{array}{l}\text { Medical history and } \\
\text { reported information }\end{array}$ & Post \\
\hline \multirow[t]{3}{*}{ Supervised exercise program } & Adherence and compliance & Attendance register & Post \\
\hline & Expectations & Open question & Post \\
\hline & Satisfaction & 9-item questionnaire & Post \\
\hline
\end{tabular}

LVEF left ventricular ejection fraction, GLS global longitudinal strain, TTE transthoracic echocardiography, CPET cardiopulmonary exercise test, NT- proBNP N-terminal brain natriuretic propeptide, BMI body mass index, BPM blood pressure monitor, SPADI shoulder pain and disability index, PREDIMED PREvención con Dleta MEDiterránea, FACT-B + 4 functional assessment of cancer therapy—breast plus arm morbidity, HADS hospital anxiety and depression scale, GLTEQ Godin Leisure Test Exercise Questionnaire, $E G$ experimental group 
tests. The CR and Preventive Cardiology specialists perform the other evaluation components, including cardiopulmonary function, physical performance and biopsychosocial aspects.

\section{Primary outcomes}

Cardiotoxicity is defined by either a significant decrease of LVEF or GLS, as previously described (9). GLS is included as it is an early and more sensitive indicator of subclinical heart failure, while a decrease in LVEF represents more advanced stages of the disease [10]. The presence of episodes of heart failure will be recorded. LVEF is calculated using Simpson's biplane rule from the apical 4- and 2-chamber view and GLS is obtained by speckletracking analysis of 4-, 3- and 2- chamber view with 2-dimensional echocardiography. Echocardiographic measurements are performed with GE Vivid E95 (GE Vingmed Ultrasound AS, Horten, Norway) ultrasound system by the same experienced operator and doubled checked by a cardiac imaging expert, both of whom are blinded to the clinical data.

A broader assessment of cardiotoxicity includes the full spectrum of cardiovascular complications of cancer treatment and considers other diagnostic options $[9,11]$, as reflected in the secondary outcomes section.

\section{Secondary outcomes}

\section{Other cardiac function outcomes}

- Cardiopulmonary function: peak VO2 (ml $/ \mathrm{kg} / \mathrm{min})$, percentage of predicted peak VO2 (\%ppVO2) and metabolic equivalents of task (METs) are assessed by CPET on a continuous incremental test in a cycle ergometer (CardioWise Ergo Fit, Pirmasens, Germany), with workload calculated for each patient depending on body mass, age and fitness.

- Biomarkers: biomarkers of myocardial damage including $\mathrm{N}$-terminal brain natriuretic propeptide (NT-proBNP) and troponin I and hemoglobin (g/dl) are determined on a blood sample.

\section{Body composition, anthropometrics and vital signs}

Body composition, including \% body fat and lean mass, are assessed by electrical bioimpedance (Tanita $M C$ 780P-MA). Anthropometrics include assessment of: height, weight, abdominal circumference and body mass index (BMI). Vital signs, including heart rate and blood pressure, are assessed with a blood pressure monitor (Omron M3 IT).

\section{Cardiovascular risk factors}

The presence of dyslipidemia, diabetes mellitus, arterial hypertension and smoking status are checked on the electronic clinical history and through clinical interview.

\section{Limb strength and upper limb function}

Lower limb strength is assessed by the number of repetitions in a 30-s sit-to-stand test; and upper limb strength (in $\mathrm{kg}$ ) is measured in both arms using handgrip dynamometry (Jamar Hydraulic Hand Dynamometer). Upper limb function evaluation in women who have undergone breast surgery or lymphadenectomy is conducted measuring upper limb perimeters for the detection of lymphedema [32]; shoulder functional capacity assessed by range of motion by goniometry [33]; and shoulder pain and disability assessed by the Shoulder Pain And Disability Index (SPADI) questionnaire [34].

\section{Psychometric status and healthy lifestyle habits}

Anxiety and depression is assessed by the Hospital Anxiety and Depression Scale (HADS) [35]. The Healthrelated quality of life by the Functional Assessment of Cancer Therapy-Breast plus Arm Morbidity (FACT$\mathrm{B}+4)$ questionnaire [36]. The level of physical activity is quantified through the Godin Leisure Test Exercise Questionnaire (GLTEQ) expressed as METs/week [37]. Adherence to Mediterranean diet with the PREDIMED (PREvención con DIeta MEDiterránea) questionnaire [38].

\section{Exploratory outcomes}

Physical exercise performed out of hospital by participants in both groups is registered in an exercise diary addressing modality (aerobic, strength, flexibility) and exercise duration (minutes). Additionally, supervised exercise performed in-hospital by participants in the EG is recorded by the physiotherapist.

Safety of the intervention is monitored by the number of adverse events occurred during supervised exercise sessions and other clinical serious adverse events (e.g., anaemia, neutropenia, episodes of heart failure) according to the Common Terminology Criteria for Adverse Events version 4.0 (CTCAE v.4.0) [39].

Adherence to the intervention is defined as the rate of patients completing the program out of those who initiate it, and compliance rate is defined by the number of exercise sessions attended divided by the number of sessions planned. Finally, program-related expectations are assessed at baseline and at the end of the program through an open question ("What do you expect 
to achieve by participating in the program?"), with responses being categorized into psychological, social and physical spheres. Satisfaction at the end of the program is evaluated by a 9-item questionnaire designed ad hoc.

\section{Data management}

Data is collected electronically through an encrypted online database designed for each study procedure (screening, initial/final assessment, etc.). No identification data is recorded in the database, but an identification number is assigned to each patient. This number is associated to the patient's clinical history number in an independent table only accessible to the ONCORE investigators on a corporate computer. All the information handled is dissociated from the patient's identification information (pseudonymized) and only ONCORE investigators will have access to the identification details.

\section{Blinding}

The type of intervention performed precludes blinding patients or staff involved. Thus, although the professionals involved in the initial assessment (before randomization) are blinded, only those who perform the echocardiogram and the registration of biomarkers are blinded in the final assessment.

\section{Sample size calculation}

Considering the main variable of analysis "development of cardiotoxicity" [9], sample size is determined by comparing two independent proportions to examine the effect of an exercise-based CR program. Based on an expected prevalence of cardiotoxicity in the CG of $20 \%$ [31] and $10 \%$ in the EG, a variation of $10 \%$ between groups is detected, that is, an expected cardiotoxicity prevention of $50 \%$ in the EG. The Marrugat et al. [40] formula was used for sample size estimation, accepting an alpha risk of 0.05 and a statistical power of $80 \%$, resulting in the need to recruit 154 participants in each arm. With an expected $10 \%$ rate of patients who will not complete the treatment or drop out, it will be necessary to include a total of 340 patients (experimental arm: $n=170$; usual care: $\mathrm{n}=170$ ).

\section{Statistical analysis}

Analyses will be conducted with SPSS v.25 and R v.4.0.2. software. Both approaches intention to treat and perprotocol analysis will be used. Descriptive analyses will comprise calculating the mean, median, standard deviation, interquartile range for quantitative variables, and absolute and relative frequencies for categorical variables. Distribution and normality of the variables will be determined by one-sample Kolmogorov-Smirnov tests for a sample (significance $<0.05$ ). To detect differences in changes between groups, the data will be analysed using Student's T or ANOVA for parametric variables and with the Wilcoxon test for non-parametric variables. Also effect size will be calculated to see the variance between both groups.

Additionally, correlation and regression analyses will be performed to determine whether the echocardiographic parameters are significantly associated with the changes in cardiopulmonary function, physical performance (e.g., muscle strength), and psychometric status (e.g., quality of life) and lifestyle habits (e.g., diet).

\section{Dissemination}

The trial results will be submitted for publication in a peer-reviewed clinical journal without restrictions and presented at relevant conferences. The investigation will be part of a doctoral thesis project signed by the first author.

\section{Discussion}

This protocol describes a randomized controlled study to examine the effect of an exercise-based CR program on the prevention of chemotherapy-induced cardiotoxicity assessed by echocardiographic parameters (LVEF and GLS), compared with conventional management. Cardiovascular disease is increasing among women with breast cancer and becoming a leading causes of death [4, 12]. Gernaat et al. [5] reported an absolute risk from 1.6 to $10.4 \%$ in breast cancer survivors. The increasing use of cardiotoxic treatments, such as anthracyclines and anti-HER2 antibodies, together with cardiovascular risk factors, are the main cause of cardiovascular health worsening. Asymptomatic cardiac dysfunction defined by a subclinical decrease of LVEF, has been associated with a higher incidence of symptomatic heart failure, which occurs in up to $20 \%$ of patients depending on treatment regimen [31]. Therefore, other parameters such as GLS, biomarkers or $\mathrm{VO} 2$ are considered to increase the sensitivity in the subclinical detection of cardiotoxicity [12].

Coordinated efforts in the early diagnosis of cardiotoxicity within $\mathrm{COH}$ units present an opportunity for the study of pharmacological and non-pharmacological strategies to prevent it. Exercise-based CR programs are a ready-to-use resource and can be a valid non-pharmacological treatment strategy, potentially extendable to cancer patients. This network highlights the need for a multidisciplinary group of professionals working in an integrated care process. Exercise-based CR programs offer a comprehensive approach, with cardiovascular risk factors management, psychosocial support and healthy lifestyle counseling. This therapy modality encourages patients to have an active role in their therapeutic 
process, contributing to increase their perception of control [41].

Exercise, pointed out as a core component of these programs, has been shown to be safe and effective in mitigating side effects of breast cancer treatment, such as worsening quality of life [21], fatigue [20] or decreased physical function [42]. However, several studies have highlighted the need for more evidence on the cardioprotective effects of exercise in preventing cardiotoxicity and improving cardiopulmonary function $[4,12,29$, 43]. Among studies applying an exercise program and evaluating heart function [43-49], broad differences are described for (1) sample size; (2) the period of application of the intervention (during or after treatment); (3) outcomes (echocardiographic parameters, peak VO2, biomarkers, cardiovascular risk factors, etc.); and (iv) evaluation methods (echocardiography, cardiopulmonary exercise testing, indirect estimation of $\mathrm{VO} 2$, blood tests, etc.). In addition, some studies [45, 50, 51] and protocols [52-54] have taken into account a broad spectrum of measurements for cardiac function assessment. Kirkham et al. [50] analysed the efficacy of a single aerobic bout performed $24 \mathrm{~h}$ prior to each anthracycline treatment in the prevention of breast cancer cardiotoxicity. These authors did not observe a relevant effect of the intervention on the reduction of subclinical cardiotoxicity (echocardiographic or circulating cardiac biomarkers outcomes) but did observe a positive systemic effect comprising hemodynamic adaptations, positive psychological effects, improved body musculoskeletal symptoms and body weight reduction. In turn, a non-randomized trial conducted by Howden et al. [12] examined the cardioprotective effect of a supervised exercise program ( 2 days/week supervised and 1 day/week unsupervised) in women with breast cancer receiving anthracyclines. They found an attenuation of the decrease in $\mathrm{VO} 2$ and higher levels of troponin in the exercise group compared to the usual care group, but no relevant changes were observed in echocardiographic outcomes. Otherwise, in a randomized controlled trial, Hojan et al. [51] compared a 5-day/week exercise program for 9 weeks with usual care in women with HER2 positive breast cancer, noting a protective effect of the exercise program on reducing LVEF and cardiopulmonary function assessed by 6 -min walking test, while on plasma parameters they did not find significant differences. Thus, the role of exercise in preventing chemotherapy-induced cardiotoxicity remains unclear. The ONCORE study attempts to elucidate how an exercise-based CR program may contribute to prevent cardiotoxicity in this setting. To this effect, this randomized study includes a larger sample of patients and performs a comprehensive assessment of cardiac function combining TTE, CPET and biomarkers, along with the monitoring of cardiovascular risk factors. In addition, the effects on psychosocial health and physical performance are recorded.

This study has limitations that should be underlined. First, although radiotherapy is also a cardiotoxic treatment [55], it has not been considered as an inclusion criterion alone. This implies that at the end of the study some patients would have received radiotherapy and others would have not. Therefore, radiotherapy sessions received by every patient are recorded for appropriate adjustments. On the other hand, as the overexpression of HER 2 occurs in approximately $15-20 \%$ of breast cancers [56], the follow-up time in the CR program may differ between patients receiving different treatment schemes, being an expected source of heterogeneity. Given the long duration of the study, especially in patients followed for more than 12 months (HER2 positive), a higher risk of dropout is expected. However, a lengthy intervention duration may benefit the physiological changes induced by exercise adaptations, reflecting a higher effect on echocardiographic and cardiopulmonary function variables. On the other hand, this trial tries to reproduce the reality of clinical practice with all its sources of heterogeneity.

Prevention of cardiotoxicity through an exercisebased CR program would have a high clinical impact $[12,29,43]$. The positive results of this study would be easily transferable to clinical practice in centers that have CR programs, establishing a new treatment indication and providing collaborative support between cardiology, oncology and rehabilitation. The proposed research question is concrete, with relevant clinical implications and achievable with low risk and reasonable investment of resources.

\section{Adaptations due to COVID-19 pandemic}

Due to the situation resulting from the Covid-19 pandemic and to give continuity to the ONCORE project, some amendments are incorporated to guarantee the safety of patients:

- Intervention in the EG: during Covid-19 pandemic, face-to-face supervised therapeutic exercise sessions had to be stopped and telematic supervised exercise sessions are proposed. Exercise in the aerobic-anaerobic transition zone performed on a cycle ergometer or treadmill would be replaced by 14 min interval circuit of 3 series of 5 exercises, with $40 \mathrm{~s}$ of work and 10 of rest. This adaptation adds $10 \mathrm{~min}$ for strengthening and mobility exercises. 
- Experimental group during the pandemic will be a subgroup that would also be analyzed independently.

- Assessment: cardiopulmonary function assessment by CPET would have to be temporarily stopped as disinfection of ventilation systems cannot be ensured. The 6-min walking test (6MWT) would be used to estimate peak VO2 [57].

- Exclusion criteria: a new exclusion criterion would be added: "Patients without possibility to join the telematic exercise sessions".

- Satisfaction questionnaire: questions related with telematic supervised exercise would be include.

\section{Trial status}

Recruitment for the study commenced in August 2018 and is expected to be completed by March 1st, 2021, considering the withdrawal of one center from the study.

\begin{abstract}
Abbreviations
BMI: Body Mass Index; BPM: Blood pressure monitor; CG: Control group; $\mathrm{COH}$ Cardio-oncohematology; CPET: Cardiopulmonary exercise test; CR: Cardiac rehabilitation; CRPCU: Cardiac rehabilitation and preventive cardiology unit; CTX: Chemotherapy; EG: Experimental group; GLS: Global longitudinal strain; GLTEQ: Godin Leisure Test Exercise Questionnaire; FACT-B + 4: Functional Assessment of Cancer Therapy — breast plus arm morbidity; HADS: Hospital Anxiety and Depression Scale; HER2: Human epidermal growth factor receptor 2; LVEF: Left ventricular ejection fraction; NT- proBNP: N-terminal brain natriuretic propeptide; PREDIMED: PREvención con Dleta MEDiterránea; SPADI: Shoulder pain and disability index; TTE: Transthoracic echocardiogram.
\end{abstract}

\section{Supplementary Information}

The online version contains supplementary material available at https://doi. org/10.1186/s12872-021-01970-2.

Additional file 1. SPIRIT 2013 checklist

\section{Acknowledgements}

The authors thank the Cardio-Onco-Hematology and the Cardiac Rehabilitation staff for their support. We specially thank M. Sestayo-Fernández for her language editing, B. Villamayor for her suggestions to the design of the training program and control of lymphedema. Assistance provided by staff of the Health Research Institute of Santiago de Compostela (IDIS) was greatly appreciated.

\section{Authors' contribution}

CPG, AMM, EDB, VGS, JRGJ contributed to the conception and design of the project. AMM, MPP, PPO, RLL coordinated and supervised the COH unit; CPG, $E D B, V G S$ designed and supervised the cardiopulmonary function assessment; EDB designed and supervised the exercise program; CPG, VGS, EDB designed the database and drafted the statistical plan; EDB, VGS, BRR drafted the manuscript, which was critically revised and approved by all authors CPG, VGS, BRR, EDB, AMM, MPP, PPO, RLL, JRGJ. All authors read and approved the final manuscript.

\section{Funding}

Financial support for this work is provided by a competitive grant of the Spanish Health Research Fund of the Carlos III Health Institute (ISCIII) - PI17/01687, co-funded FEDER, through Strategic Action in Healthcare, 2017. The ISCIII had no role in study design, data collection, data analysis, data interpretation, or writing of the report. The ISCIII is the national and international reference in biomedical research and public health in Spain. This is the Government's Public Research Organisation and its role is to promote research and innovation in Health Sciences and Biomedicine, and to provide scientific-technical reference services and teaching programmes for the National Health System.

\section{Availability of data and materials}

Data are available upon reasonable request.

\section{Declarations}

\section{Ethics approval and consent to participate}

This study has the approval of the local ethics committee (Ethics Committee of Clinical Investigation of Galicia; reference number 2018/083) on May 29, 2018 and any proposed protocol change will be submitted for a new review and approval. This project also comprises with the principles of the Declaration of Helsinki. All participants will provide written informed consent to participate in the study.

\section{Consent for publication}

Not applicable.

\section{Competing interests}

The authors have no conflicts of interest to disclose.

\section{Author details}

${ }^{1}$ Cardiology Department, Centro de Investigación Biomédica en Red de Enfermedades Cardiovasculares (CIBERCV), University Clinical Hospital of Santiago de Compostela (SERGAS), A Choupana s/n, 15706 Santiago de Compostela, A Coruña, Spain. ${ }^{2}$ Health Research Institute of Santiago de Compostela (IDIS), A Coruña, Spain. ${ }^{3}$ Department of Physiotherapy, Medicine and Biomedical Sciences, Faculty of Physiotherapy, University of A Coruña, A Coruña, Spain. ${ }^{4}$ Psychosocial Intervention and Functional Rehabilitation Research Group, Department of Physiotherapy, Medicine and Biomedical Sciences, Faculty of Physiotherapy, University of A Coruña, A Coruña, Spain. ${ }^{5}$ Medical Oncology Department and Translational Medical Oncology Group, Centro de Investigación Biomédica en Red de Cáncer (CIBERONC), Santiago de Compostela University School of Medicine, University Clinical Hospital of Santiago (SERGAS), Santiago de Compostela, A Coruña, Spain.

Received: 10 February 2021 Accepted: 29 March 2021

Published online: 07 April 2021

\section{References}

1. Siegel RL, Miller KD, Jemal A. Cancer statistics, 2020. CA Cancer J Clin. 2020;70:7-30. https://doi.org/10.3322/caac.21590.

2. Zamorano JL, Lancellotti P, Rodriguez Muñoz D, Aboyans V, Asteggiano R, Galderisi M, et al. 2016 ESC Position Paper on cancer treatments and cardiovascular toxicity developed under the auspices of the ESC Committee for Practice Guidelines. Eur J Heart Fail. 2017;19:9-42. https://doi.org/ 10.1002/ejhf.654.

3. Yeh ETH. Cardiotoxicity induced by chemotherapy and antibody therapy. Annu Rev Med. 2006;57:485-98. https://doi.org/10.1146/annurev.med.57. 121304.131240.

4. Kirkham AA, Beaudry RI, Paterson DI, Mackey JR, Haykowsky MJ. Curing breast cancer and killing the heart: a novel model to explain elevated cardiovascular disease and mortality risk among women with early stage breast cancer. Prog Cardiovasc Dis. 2019;62:1 16-26. https://doi.org/10. 1016/j.pcad.2019.02.002.

5. Gernaat SAM, Ho PJ, Rijnberg N, Emaus MJ, Baak LM, Hartman M, et al. Risk of death from cardiovascular disease following breast cancer: a systematic review. Breast Cancer Res Treat. 2017;164:537-55. https://doi. org/10.1007/s10549-017-4282-9.

6. Bradshaw PT, Stevens J, Khankari N, Teitelbaum SL, Neugut Al, Gammon MD. Cardiovascular disease mortality among breast cancer survivors. Epidemiology. 2016;27:6-13. https://doi.org/10.1097/EDE.0000000000 000394. 
7. Ezaz G, Long JB, Gross CP, Chen J. Risk prediction model for heart failure and cardiomyopathy after adjuvant trastuzumab therapy for breast cancer. J Am Heart Assoc. 2014;3:e000472. https://doi.org/10.1161/JAHA. 113.000472.

8. Slamon D, Eiermann W, Robert N, Pienkowski T, Martin M, Press M, et al. Adjuvant Trastuzumab in HER2-positive breast cancer. N Engl J Med. 2011;365:1273-83. https://doi.org/10.1056/NEJMoa0910383.

9. Plana JC, Galderisi M, Barac A, Ewer MS, Ky B, Scherrer-Crosbie M, et al. Expert consensus for multimodality imaging evaluation of adult patients during and after cancer therapy: a report from the American Society of Echocardiography and the European Association of Cardiovascular Imaging. Eur Heart J Cardiovasc Imaging. 2014;15:1063-93. https://doi.org/10. 1093/ehjci/jeu192.

10. Thavendiranathan P, Poulin F, Lim KD, Plana JC, Woo A, Marwick TH. Use of myocardial strain imaging by echocardiography for the early detection of cardiotoxicity in patients during and after cancer chemotherapy: a systematic review. J Am Coll Cardiol. 2014;63(25):2751-68. https://doi. org/10.1016/j.jacc.2014.01.073.

11. Lenneman CG, Sawyer DB. Cardio-oncology: an update on cardiotoxicity of cancer-related treatment. Circ Res. 2016;118:1008-20. https://doi.org/ 10.1161/CIRCRESAHA.115.303633.

12. Scott JM, Nilsen TS, Gupta D, Jones LW. Exercise therapy and cardiovascular toxicity in cancer. Circulation. 2018;137:1176-91. https://doi.org/10. 1161/CIRCULATIONAHA.117.024671.

13. Armenian SH, Lacchetti C, Barac A, Carver J, Constine LS, Denduluri N, et al. Prevention and monitoring of cardiac dysfunction in survivors of adult cancers: American society of clinical oncology clinical practice guideline. J Clin Oncol. 2017;35:893-911. https://doi.org/10.1200/JCO. 2016.70.5400.

14. Kirkham AA, Virani SA, Campbell KL. The utility of cardiac stress testing for detection of cardiovascular disease in breast cancer survivors: a systematic review. Int J Womens Health. 2015;7:127-40. https://doi.org/10.2147/ IJWH.S68745.

15. Barish R, Lynce F, Unger K, Barac A. Management of cardiovascular disease in women with breast cancer. Circulation. 2019;139:1110-20. https:// doi.org/10.1161/CIRCULATIONAHA.118.039371.

16. Campbell KL, Winters-Stone KM, Wiskemann J, May AM, Schwartz AL, Courneya KS, et al. Exercise guidelines for cancer survivors: consensus statement from international multidisciplinary roundtable. Med Sci Sports Exerc. 2019;51:2375-90. https://doi.org/10.1249/MSS.0000000000 002116.

17. Segal R, Zwaal C, Green E, Tomasone JR, Loblaw A, Petrella T, et al. Exercise for people with cancer: a clinical practice guideline. Curr Oncol. 2017;24:40-6. https://doi.org/10.3747/co.24.3376.

18. Cormie P, Zopf EM, Zhang X, Schmitz KH. The impact of exercise on cancer mortality, recurrence, and treatment-related adverse effects. Epidemiol Rev. 2017;39:71-92. https://doi.org/10.1093/epirev/mxx007.

19. Mustian KM, Sprod LK, Janelsins M, Peppone LJ, Mohile S. Exercise recommendations for cancer-related fatigue, cognitive impairment, sleep problems, depression, pain, anxiety, and physical dysfunction - a review. Oncol Hematol Rev. 2012;08:81. https://doi.org/10.17925/OHR.2012.08.2. 81.

20. Meneses-Echávez JF, González-Jiménez E, Ramírez-Vélez R. Effects of supervised exercise on cancer-related fatigue in breast cancer survivors: a systematic review and meta-analysis. BMC Cancer. 2015. https://doi.org/ 10.1186/s12885-015-1069-4.

21. Hong F, Ye W, Kuo CH, Zhang Y, Qian Y, Korivi M. Exercise intervention improves clinical outcomes, but the "time of session" is crucial for better quality of life in breast cancer survivors: a systematic review and metaanalysis. Cancers. 2019. https://doi.org/10.3390/cancers11050706.

22. Yu AF, Jones LW. Breast cancer treatment-associated cardiovascular toxicity and effects of exercise countermeasures. Cardio-Oncol. 2016;2:1. https://doi.org/10.1186/s40959-016-0011-5.

23. Jones LW, Habel LA, Weltzien E, Castillo A, Gupta D, Kroenke CH, et al. Exercise and risk of cardiovascular events in women with nonmetastatic breast cancer. J Clin Oncol. 2016;34:2743-9. https://doi.org/10.1200/JCO. 2015.65.6603.

24. Ginzac A, Passildas J, Gadéa E, Abrial C, Molnar I, Trésorier R, et al. Treatment-induced cardiotoxicity in breast cancer: a review of the interest of practicing a physical activity. Oncology (Switzerland). 2019;96:223-34. https://doi.org/10.1159/000499383.
25. McMahon SR, Ades PA, Thompson PD. The role of cardiac rehabilitation in patients with heart disease. Trends Cardiovasc Med. 2017;27:420-5. https://doi.org/10.1016/j.tcm.2017.02.005.

26. Corrà U, Piepoli MF, Carré F, Heuschmann P, Hoffmann U, Verschuren $M$, et al. Secondary prevention through cardiac rehabilitation: physical activity counselling and exercise training: key components of the position paper from the Cardiac Rehabilitation Section of the European Association of Cardiovascular Prevention and Rehabilitat. Eur Heart J. 2010;31:1967-74. https://doi.org/10.1093/eurheartj/ehq236.

27. Rauch B, Davos CH, Doherty P, Saure D, Metzendorf MI, Salzwedel A, et al. The prognostic effect of cardiac rehabilitation in the era of acute revascularisation and statin therapy: A systematic review and meta-analysis of randomized and non-randomized studies-The Cardiac Rehabilitation Outcome Study (CROS). Eur J Prev Cardiol. 2016;23:1914-39. https://doi. org/10.1177/2047487316671181.

28. Ponikowski P, Voors AA, Anker SD, Bueno H, Cleland JGF, Coats AJS, et al. 2016 ESC Guidelines for the diagnosis and treatment of acute and chronic heart failure. Eur Heart J. 2016;37:2129-2200m. https://doi.org/10. 1093/eurheartj/ehw128.

29. Venturini E, lannuzzo G, D'Andrea A, Pacileo M, Tarantini L, Canale ML, et al. Oncology and cardiac rehabilitation: an underrated relationship. J Clin Med. 2020;9:1810. https://doi.org/10.3390/jcm9061810.

30. Chan AW, Tetzlaff JM, Altman DG, Laupacis A, Gøtzsche PC, Krle A-Jerić K, SPIRIT, , et al. Statement: defining standard protocol items for clinical trials. Rev Panam Salud Publica. 2013;2015(38):506-14. https://doi.org/10. 7326/0003-4819-158-3-201302050-00583.

31. López-Fernández T, Thavendiranathan P. Emerging cardiac imaging modalities for the early detection of cardiotoxicity due to anticancer therapies. Rev Española Cardiol (English Ed). 2017;70:487-95. https://doi. org/10.1016/j.rec.2017.01.004.

32. Fu MR. Breast cancer-related lymphedema: symptoms, diagnosis, risk reduction, and management. World J Clin Oncol. 2014;5:241-7. https:// doi.org/10.5306/wjco.v5.i3.241.

33. Norkin CC, White DJ. Measurement of joint motion: a guide to goniometry. Philadelphia: FA Davis; 2016.

34. Torres-Lacomba M, Sánchez-Sánchez B, Prieto-Gómez V, Pacheco-daCosta S, Yuste-Sánchez MJ, Navarro-Brazález B, et al. Spanish cultural adaptation and validation of the shoulder pain and disability index, and the oxford shoulder score after breast cancer surgery. Health Qual Life Outcomes. 2015;13:63. https://doi.org/10.1186/s12955-015-0256-y.

35. Herrero MJ, Blanch J, Peri JM, De Pablo J, Pintor L, Bulbena A. A validation study of the hospital anxiety and depression scale (HADS) in a Spanish population. Gen Hosp Psychiatry. 2003;25:277-83. https://doi.org/10. 1016/S0163-8343(03)00043-4.

36. Brady MJ, Cella DF, Mo F, Bonomi AE, Tulsky DS, Lloyd SR, et al. Reliability and validity of the functional assessment of cancer therapy- breast quality-of-life instrument. J Clin Oncol. 1997;15:974-86. https://doi.org/ 10.1200/JCO.1997.15.3.974.

37. Amireault S, Godin G, Lacombe J, Sabiston CM. Validation of the Godinshephard leisure-time physical activity questionnaire classification coding system using accelerometer assessment among breast cancer survivors. J Cancer Surviv. 2015;9:532-40. https://doi.org/10.1007/ s11764-015-0430-6.

38. Panagiotakos DB, Pitsavos C, Stefanadis C. Dietary patterns: a Mediterranean diet score and its relation to clinical and biological markers of cardiovascular disease risk. Nutr Metab Cardiovasc Dis. 2006;16:559-68. https://doi.org/10.1016/j.numecd.2005.08.006.

39. National Cancer Institute. Common Terminology Criteria for Adverse Events (CTCAE). Cancer Therapy Evaluation Program. https://ctep.cancer. gov/protocoldevelopment/electronic_applications/ctc.htm. Accessed 28 Dec 2020.

40. Marrugat J, Vila J, Pavesi M, Sanz F. Estimación del tamaño de la muestra en la investigación clínica y epidemiológica. Med Clin. 1998;111:267-76.

41. Dolan LB, Barry D, Petrella T, Davey L, Minnes A, Yantzi A, et al. The cardiac rehabilitation model improves fitness, quality of life, and depression in breast cancer survivors. J Cardiopulm Rehabil Prev. 2018;38:246-52. https://doi.org/10.1097/HCR.0000000000000256.

42. Kirkham AA, Bland KA, Sayyari S, Campbell KL, Davis MK. Clinically relevant physical benefits of exercise interventions in breast cancer survivors. Curr Oncol Rep. 2016;18:12. https://doi.org/10.1007/s11912-015-0496-3. 
43. Scott JM, Thomas SM, Peppercorn JM, Herndon JE, Douglas PS, Khouri $M G$, et al. Effects of exercise therapy dosing schedule on impaired cardiorespiratory fitness in patients with primary breast cancer: a randomized controlled trial. Circulation. 2020;141:560-70. https://doi.org/10.1161/ CIRCULATIONAHA.119.043483.

44. Scott JM, Zabor EC, Schwitzer E, Koelwyn GJ, Adams SC, Nilsen TS, et al. Efficacy of exercise therapy on cardiorespiratory fitness in patients with cancer: a systematic review and meta-analysis. J Clin Oncol. 2018:36:2297-305. https://doi.org/10.1200/JCO.2017.77.5809.

45. Howden EJ, Bigaran A, Beaudry R, Fraser S, Selig S, Foulkes S, et al. Exercise as a diagnostic and therapeutic tool for the prevention of cardiovascular dysfunction in breast cancer patients. Eur J Prev Cardiol. 2019;26:305-15. https://doi.org/10.1177/2047487318811181.

46. Casla S, López-Tarruella S, Jerez Y, Marquez-Rodas I, Galvão DA, Newton $\mathrm{RU}$, et al. Supervised physical exercise improves VO2max, quality of life, and health in early stage breast cancer patients: a randomized controlled trial. Breast Cancer Res Treat. 2015;153:371-82. https://doi.org/10.1007/ s10549-015-3541-x.

47. Maginador G, Lixandrão ME, Bortolozo HI, Vechin FC, Sarian LO, Derchain $\mathrm{S}$, et al. Aerobic exercise-induced changes in cardiorespiratory fitness in breast cancer patients receiving chemotherapy: A systematic review and meta-analysis. Cancers. 2020;12:1-14. https://doi.org/10.3390/cancers120 82240.

48. Kirkham AA, Shave RE, Bland KA, Bovard JM, Eves ND, Gelmon KA, et al. Protective effects of acute exercise prior to doxorubicin on cardiac function of breast cancer patients: a proof-of-concept RCT. Int J Cardiol. 2017;245:263-70. https://doi.org/10.1016/j.ijcard.2017.07.037.

49. Courneya KS, Mackey JR, Bell GJ, Jones LW, Field CJ, Fairey AS. Randomized controlled trial of exercise training in postmenopausal breast cancer survivors: Cardiopulmonary and quality of life outcomes. J Clin Oncol. 2003:21:1660-8. https://doi.org/10.1200/JCO.2003.04.093.

50. Kirkham AA, Eves ND, Shave RE, Bland KA, Bovard J, Gelmon KA, et al. The effect of an aerobic exercise bout $24 \mathrm{~h}$ prior to each doxorubicin treatment for breast cancer on markers of cardiotoxicity and treatment symptoms: a. Breast Cancer Res Treat. 2018;167:719-29. https://doi.org/ 10.1007/s10549-017-4554-4.

51. Hojan K, Procyk D, Horyńska-Kęstowicz D, Leporowska E, Litwiniuk M. The Preventive role of regular physical training in ventricular remodeling, serum cardiac markers, and exercise performance changes in breast cancer in women undergoing trastuzumab therapy-an REH-HER study. J Clin Med. 2020;9:1379. https://doi.org/10.3390/jcm9051379.

52. Jacquinot $Q$, Meneveau N, Chatot M, Bonnetain F, Degano B, Bouhaddi M et al. A phase 2 randomized trial to evaluate the impact of a supervised exercise program on cardiotoxicity at 3 months in patients with HER2 overexpressing breast cancer undergoing adjuvant treatment by trastuzumab: design of the CARDAPAC study. BMC Cancer. 2017;17:425. https:// doi.org/10.1186/s12885-017-3420-4

53. Antunes P, Esteves D, Nunes C, Sampaio F, Ascensão A, Vilela E, et al. Impact of exercise training on cardiotoxicity and cardiac health outcomes in women with breast cancer anthracycline chemotherapy: a study protocol for a randomized controlled trial. Trials. 2019;20:433. https://doi. org/10.1186/s13063-019-3499-9.

54. Kirkham AA, Paterson DI, Prado CM, Mackey JM, Courneya KS, Pituskin E, et al. Rationale and design of the caloric restriction and exercise protection from anthracycline toxic effects (CREATE) study: a 3-arm parallel group phase II randomized controlled trial in early breast cancer. BMC Cancer. 2018;18:864. https://doi.org/10.1186/s12885-018-4778-7.

55. Walker V, Jacob S, Crijns A, Langendijk J, Spoor D, Vliegenthart R, et al. Radiotherapy-induced cardiotoxicity in breast cancer patients: New approaches of early detection and prediction based on cardiac imaging and circulating biomarkers (European MEDIRAD EARLY-HEART study). Arch Cardiovasc Dis Suppl. 2019;11:124-5. https://doi.org/10.1016/j. acvdsp.2018.10.275

56. Burstein $\mathrm{HJ}$. The distinctive nature of HER2-positive breast cancers. N Engl J Med. 2005;353:1652-4. https://doi.org/10.1056/NEJMp058197.

57. Schumacher AN, Shackelford DYK, Brown JM, Hayward R. Validation of the 6-min walk test for predicting peak VO2 in cancer survivors. Med Sci Sports Exerc. 2019;51:271-7. https://doi.org/10.1249/MSS.0000000000 001790.

\section{Publisher's Note}

Springer Nature remains neutral with regard to jurisdictional claims in published maps and institutional affiliations.

Ready to submit your research? Choose BMC and benefit from:

- fast, convenient online submission

- thorough peer review by experienced researchers in your field

- rapid publication on acceptance

- support for research data, including large and complex data types

- gold Open Access which fosters wider collaboration and increased citations

- maximum visibility for your research: over $100 \mathrm{M}$ website views per year

At BMC, research is always in progress.

Learn more biomedcentral.com/submissions 